\title{
Preference-driven Interactive Ranking System for Personalized Decision Support
}

\author{
Caitlin Kuhlman, MaryAnn VanValkenburg, Diana Doherty, Malika Nurbekova, \\ Goutham Deva, Zarni Phyo, Elke Rundensteiner, Lane Harrison \\ cakuhlman|mevanvalkenburg|ddoherty2|mnurbekova|godeva|zphyo|rundenst|ltharrison@wpi.edu \\ Worcester Polytechnic Institute
}

\begin{abstract}
Manually constructing rankings is a tedious ad-hoc process, requiring extensive user effort to evaluate data attribute importance, and often leading to undesirable results. Meanwhile, sophisticated learning-to-rank algorithms are able to leverage large amounts of data to generate high quality rankings automatically. In this work we present RanKit, a novel technology that brings the power of automatic learning-to-rank to the public. RanKit serves as a personal recommender system for building rankings from partial user knowledge in the form of item preferences. A user-friendly rank building interface provides rich input modes for preference specification. Visual feedback on the quality of the learned ranking model is given in real time, empowering the user to guide the underlying learn-to-rank algorithm. Users are actively involved with every step of the rank generation process, developing trust in the model and producing personalized rankings suitable for real-world decision making. In this demonstration, the audience works directly with the RanKit system on public domain datasets ranging from college rankings and economic indicators to movies and sports.
\end{abstract}

\section{CCS CONCEPTS}

- Information systems $\rightarrow$ Decision support systems;

\section{KEYWORDS}

Interactive Ranking; Visual Analytics

\section{ACM Reference Format:}

Caitlin Kuhlman, MaryAnn VanValkenburg, Diana Doherty, Malika Nurbekova, Goutham Deva, Zarni Phyo, Elke Rundensteiner, Lane Harrison. 2018. Preferencedriven Interactive Ranking System for Personalized Decision Support. In The 27th ACM International Conference on Information and Knowledge Management (CIKM '18), October 22-26, 2018, Torino, Italy. ACM, New York, NY, USA, 4 pages. https://doi.org/10.1145/3269206.3269227

\section{INTRODUCTION}

Motivation. Ranking is a common technique used to discern the relative merits of objects, in particular when choosing the most valuable item or allotting resources according to position. For this important decision making strategy, an individual will typically

Permission to make digital or hard copies of all or part of this work for personal or classroom use is granted without fee provided that copies are not made or distributed for profit or commercial advantage and that copies bear this notice and the full citation on the first page. Copyrights for components of this work owned by others than ACM must be honored. Abstracting with credit is permitted. To copy otherwise, or republish, to post on servers or to redistribute to lists, requires prior specific permission and/or a fee. Request permissions from permissions@acm.org.

CIKM '18, October 22-26, 2018, Torino, Italy

(c) 2018 Association for Computing Machinery.

ACM ISBN 978-1-4503-6014-2/18/10 . \$ \$15.00

https://doi.org/10.1145/3269206.3269227 have to weigh many different attributes manually, consider which are most important to their choice, and combine them together in some ad-hoc fashion to create a ranking. This process is tedious and can be error prone. It is so difficult that we often turn to outside organizations to provide rankings for common choices - such as college rankings when we apply to school or movie or restaurant rankings when we look for entertainment. Policy makers turn to rankings of hospitals and schools when making funding decisions and business leaders use economic rankings to evaluate cities or states in which to open new companies. Proprietary rankings can possibly be misleading when the underlying ranking models are not published, or worse, they may encode negative stereotypes [8]. Furthermore, they are static, often over-generalizing without personalization for consumers with different values.

Use of rankings in recommendation and search has led to the development of many powerful learning-to-rank algorithms, capable of ranking huge datasets with many attributes automatically [6]. We encounter these machine learning-driven rankings when we search or shop online. Yet, these methods are out of reach of most people for their own personal decision making. Systems for automatic ranking are necessary to empower people to make better, more informed choices.

State-of-the-Art. To address this problem, systems have been proposed to help users more easily create rankings by manually weighting attributes and then visualizing the results $[3,5,7]$. The Lineup system [3] allows users to see how each data attribute contributes to the overall ranking. The data is displayed in a table, and by dragging the widths of the columns, users can see how the weights of the attributes impact the overall ranking. These visualizations shed much needed light on the process of designing rankings. However they still depend on manual adjustments, leaving the user to try all weight combinations to find the best result. Podium [7] is a recently proposed application where a default attribute weighting model gives an initial ranking. Thereafter, users can improve the ranking by prioritizing or devaluing specific items using visual interactions. While extremely useful, the initial ordering of all items in a ranked list risks biasing the later rank building process.

Challenges. Tools for personalized ranking must fully address the needs of decision makers including: (1) Manually deciding the importance of data attributes is an unreasonable task for a human analyst. It requires extensive knowledge of the data domain, and the infinite number of possible weight combinations is intractable, especially when the number of attributes is high. A rich dataset should be a virtue when making well-informed decisions, and such a process makes it a curse for the analyst to deal with. (2) People are not good at assigning values to individual items or attributes when faced with a large dataset. We are cognitively more adept 
at specifying preferences between items [1]. This ability should be leveraged to best incorporate the user's knowledge into the ranking process. (3) A ranking system should be an impartial tool. Previous systems initially display the data in a pre-ranked order, which unfortunately may bias users toward items displayed at the top of the list [4]. (4) Opacity prevents user trust [2]. To best incorporate automation into a visual analytics system the impact of user interactions on the underlying machine learning mechanism should accurately reflect user intention, and the process should lead to transparent interpretable results which users can employ in the real world.

The RanKit Approach. RanKit is an interactive system that transforms what had once been a difficult task of weighing the importance of many different factors to a simple process of making comparisons among a few items. The challenges above are addressed in the following ways: (1) Rankit allows users to leverage their intuition or partial understanding of a complex dataset to extract a global ranking model. (2) Multiple preference input modes (i.e., list, pairwise, and categorical comparison) allow the user to choose the representation of their preferences that best matches their mental model, capturing their knowledge in an intuitive manner. (3) Careful visual interface design avoids biasing the user to any pre-specified order of items, ensuring the resulting ranking is driven purely from user preferences. A separate view provides detailed information about the resulting global ranking. (4) The system not only learns relationships among the data points automatically, but it does so in a way that is transparent to the user. Visual feedback communicates the impact of user interactions in real time, allowing the user to drive the rank learning process by changing their input or specifying additional preferences. This empowers the user to understand and gain insight from the interplay of their item preferences and the underlying data attributes, leading to rankings which can be trusted for real-world decision making. In this demonstration we will allow users to experience this technology applied to real data sets of interest.

\section{THE RANKIT SYSTEM}

The RanKit system overview is depicted in Figure 1. Datasets to be analyzed are imported and preprocessed to clean missing values, encode categorical attributes, and normalize the data, before being housed in the RanKit Data Store. Plug-and-play design allows for any pairwise learning-to-rank algorithm to power the backend Ranking Engine. User preferences are input to the Visual Analytics layer (top of figure) using 3 intuitive comparison modes in the Build view. From these entries, pairwise relationships between items are extracted and sent to the machine learning algorithm at the Execution layer (middle of figure). The weights of the ranking model are computed incrementally with each change in the build view. In addition, diagnostic metrics are continually computed and sent back to the analytics layer to provide the user with feedback on the performance of the ranking model. Explanatory features throughout the system visually encode these confidence values. At any time, the user can switch to the Explore view, triggering ranking predictions to be made and the global ranking over all items to be sent to the front end for display.

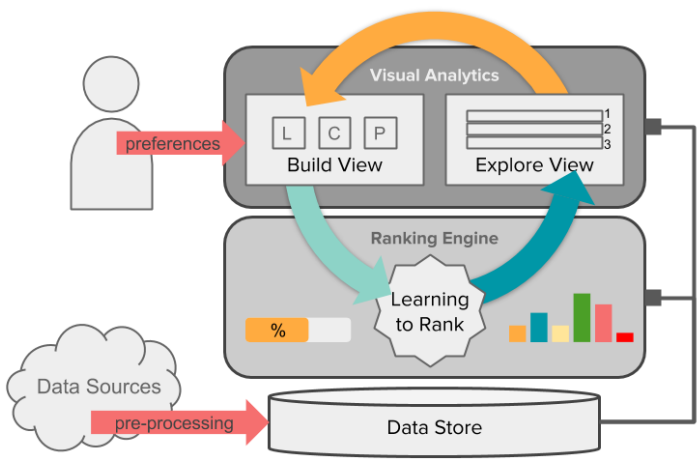

Figure 1: RanKit system overview.

\section{KEY INNOVATIONS}

\subsection{Learning from unbiased user preferences}

RanKit produces high quality rankings by employing a pairwise learning-to-rank algorithm, which reduces the problem of ranking a set of objects to a binary classification task over ordered pairs of items [6]. The pairwise problem formulation aligns nicely with user preferences for some items as opposed to others. We design three comparison modes to allow users to specify their preferences in different ways. These interfaces effectively capture user intentions, without requiring undue effort. Further, this preference specification step is separated from any ordered display of the data, to avoid predisposing the user toward biased choices.

The Pairwise Comparison mode lets users directly express their preferences as binary relations between pairs of items. For $m$ items added to the comparison tool, $m / 2$ pairs are generated. This tool is easy to understand, however specifying many pairs may be tedious. In the List Comparison mode, preferences are specified by choosing a subset of items and arranging them in an ordered list. Items at the top of the list are preferred to those below. For $m$ items added to the list, the $\left(\begin{array}{c}m \\ 2\end{array}\right)=m(m-1) / 2$ possible ordered pairs can be extracted. As a third option, in the Categorical Comparison items are binned into three possible categories: high, medium, low. Using this mode, the user doesn't have to specify relationships among items it may be unsure of - since the items within a single category are not compared - but can still indicate preferences between others. All items in one category are preferred to the items in lower categories. The number of pairs generated in this mode varies, since a different number of items can be added to each of the 3 bins. In the worst case, if all items except one are placed in a single bin, only $m-1$ pairs are generated. However if we consider an equal distribution of $m / 3$ objects in each bin, $\frac{m^{2}}{3}$ pairs can be formed.

\subsection{Facilitating real-time insights using incremental machine learning}

The set of pairs extracted from the user preferences specified via the comparison tool provide training data for the machine learning algorithm. Labels are assigned to each pair of items to indicate an order between them. For instance, given a pair $(a, b)$, label 1 is assigned if $a$ is preferred to $b$, or label -1 otherwise. A classifier is then trained to distinguish between correctly and incorrectly 


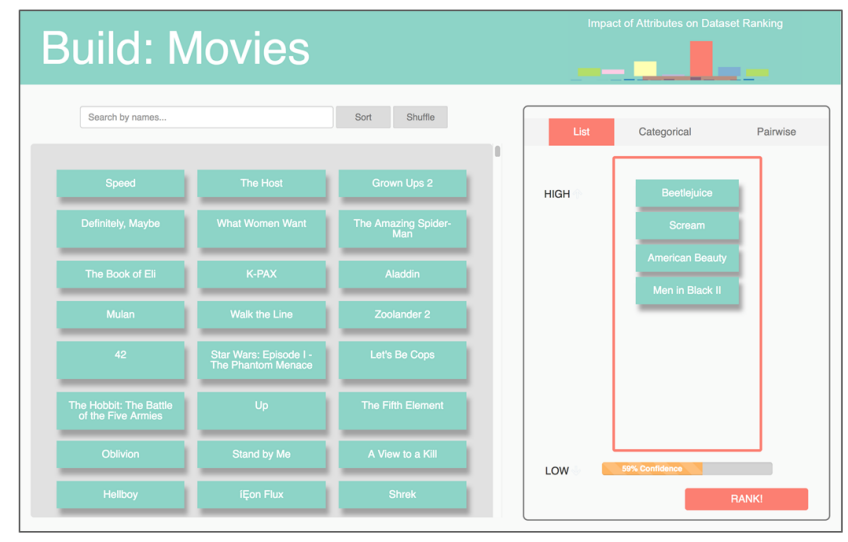

Figure 2: The RanKit Build view consists of the data pool on the left and comparison interface on the right. Preferences over the Movies dataset are shown in List Comparison mode.

ordered pairs. Many pairwise learning-to-rank algorithms exist [6] which use different methods for this classification task. RanKit features a flexible design, in that any such algorithm can be plugged in to the ranking engine. For the current technology, we choose RankSVM [4] which learns a support vector classifier to distinguish between ordered pairs of items. Since this large margin classification technique depends only on points close to the decision boundary, it is possible to learn the model incrementally. This allows for fast updates to the model, even for large datasets, to provide real-time feedback on model quality.

\subsection{Measuring uncertainty to foster user trust and understanding}

To measure the user's progress and evaluate the quality of the ranking model we design confidence metrics which are continually updated and visualized for the user. In turn, the resulting global ranking will match their expectation, and produce a model they trust. To evaluate the overall expected quality of the ranking model, we measure the number of concordant and discordant pairs predicted for the training dataset, adjusted by an estimate of a sufficient minimum number of training pairs. This confidence score is displayed as a progress bar in the Build view, instantly communicating to the user that as they add more data, the quality of the model improves. This encourages interaction in this crucial build stage to ensure that the resulting model will be able to distinguish between items in the dataset in a meaningful way.

In addition to measuring overall model quality, we design a metric to evaluate the predictive ability of the model for each individual item in the dataset. In RankSVM, the most ambiguous pairs of items are those closest to the decision boundary. Therefore, to derive a score for each item, we aggregate the distances to the boundary over all pairs it appears in. If the pairs containing a particular item tend to be far from the decision bound, then it will have a high confidence value. If many pairs containing the item are close to the decision boundary, this means its rank is difficult to distinguish from many other items. Therefore the confidence of the prediction will be low. Individual item confidence scoring allows the user to
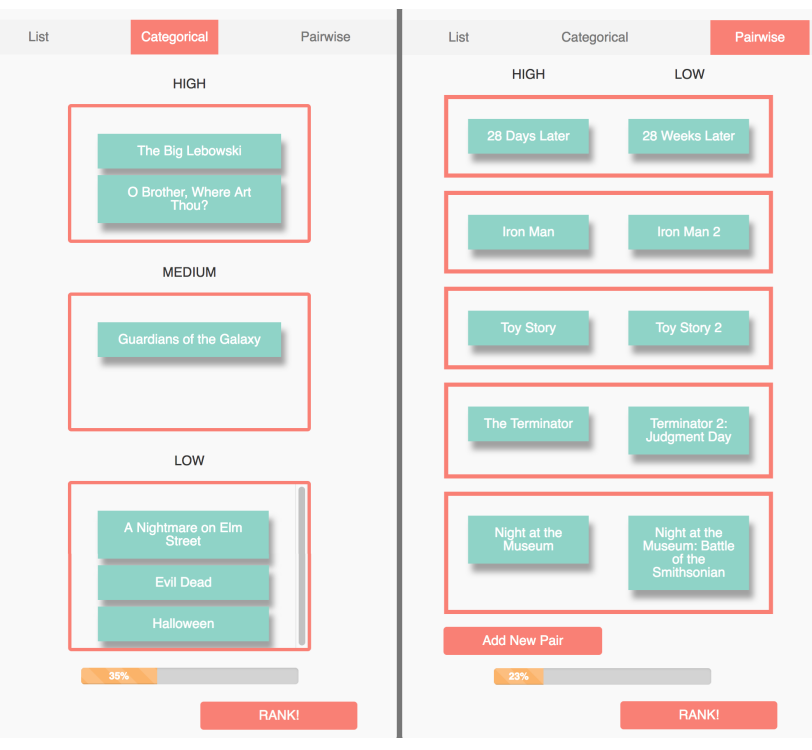

Figure 3: Preferences over items from the Movies dataset are shown in two alternative comparison modes: Categorical on the left and Pairwise on the right.

identify items which may be useful to enter in the build view to provide more information to the ranking engine.

\section{RANKIT DEMONSTRATION}

In our demonstration, the CIKM audience will directly interact with RanKit through a visual web interface (http://rankit.wpi.edu). Several real datasets from diverse domains are available for analysis, including colleges ${ }^{12}$, movies ${ }^{3}$, games ${ }^{45}$, sports ${ }^{6}$, and the US economy ${ }^{7}$. We now use the following case study on college ranking to illustrate the capabilities of the RanKit system.

Choosing a dataset and comparison method. Alice is a high school junior deciding where to apply for college. She has toured several schools around her home state. Some made a good impression while others were definitely not for her. She also has a couple of "dream" schools that she would love to attend. Given this partial understanding of a few colleges, Alice would like to know how her other potential choices stack up. She uses RanKit to analyze the Colleges dataset containing both numeric and categorical attributes of US colleges including the size, cost of tuition, geographic region, and acceptance rate. Alice selects the Colleges dataset to load and display the cleaned data in the Build view.

Entering preferences in the Build tool. Figure 2 shows the Build view layout. Colleges to be ranked are displayed on the lefthand side of the screen in a grid, with items arranged randomly. This avoids implying preference according to numeric or lexicographic order. Data attributes for each college can be accessed as a tooltip.

\footnotetext{
${ }^{1}$ https://collegescorecard.ed.gov/

${ }^{2}$ https://www.usnews.com/best-colleges/rankings/national-universities

${ }^{3}$ https://www.kaggle.com/rounakbanik/the-movies-dataset

${ }^{4}$ https://www.kaggle.com/rush4ratio/video-game-sales-with-ratings

${ }^{5}$ https://www.kaggle.com/mrpantherson/board-game-data

${ }^{6} \mathrm{https}: / /$ www.sports-reference.com/cfb/

${ }^{7}$ http://matters.mhtc.org/
} 
On the right side of the Build view, Alice has a choice of three preference collection formats. The alternative modes are shown in Figure 3. Alice decides that Categorical Comparison mode is the easiest way to enter her preferences among the colleges she is familiar with. She uses the search box to find her "dream colleges" in the data pool and drags them to the high category. She puts the schools she dislikes in the low category. She can move as many items as desired, swapping their order and moving them between the pool and the different fields within the comparison tool.

Once Alice enters items into two different categories, reactive visualizations begin showing the incremental progress of the ranking engine. The model weights appear as a bar graph in the header of the page, changing in real time to show the impact of item preferences on the learned importance of the attributes. In addition, an overall confidence score for the model is shown in a progress bar below the comparison tool. At this point, the progress bar shows a low confidence score meaning that no reliable rankings can be generated using her current input preferences.

Transitioning from the Build view to the Explore view. Impatient to see results, Alice hits the "Rank" button and is redirected to the "Explore" view. The global ranking over all colleges is displayed in a table, along with data attribute values. Several visual encodings communicate information learned from the interactions in the previous view. Color is used to visually associate the column headers with the attribute weights in the bar graph at the top of the page. Items entered in the Build view are highlighted with bold text. The overall score assigned to each item is indicated with gray horizontal bars (the numeric score is available on hover). These scores are determined by a weighted combination of the data attributes according to the learned model. In addition, a confidence value is determined for each item. This is visualized using the background coloring of the first columns of the table, with darker colors indicating higher confidence (the exact confidence score can be accessed as a tooltip). At a glance, Alice finds that none of the colleges listed at the top of the ranking have high confidence values. This indicates that she has not yet entered sufficient information. Alice thus decides to return to the Build view to improve her model.

Using feedback to improve ranking. This time, Alice adds the colleges that she visited and liked into the medium category. She notices that the confidence value in the progress bar goes up. Alice uses the "Shuffle" button to permute the items in the data pool to browse the rest of the colleges. Reflecting on what her goals are for college, Alice decides to add colleges with strong Science programs to the High category, and some without to the Low category. Continuing this way, Alice drags colleges into the comparison interface until the progress bar reaches $99 \%$. Switching back to the Explore view, Alice sees the ranking shown in Figure 4.

Understanding the ranking model. This time, Alice is pleased to see several of her favorite colleges are at the top of the ranking, as well as some she hadn't considered. The confidence values for these individual items are higher now. Alice hovers over the bar graph in the top right corner to see which attributes contribute the most to her model. She sees that enrollment is a significant contributor to the ranking, and observes that the colleges she prefers have a tendency to be large research universities.

Making a decision from the data. Looking at her ranking, two colleges that are unfamiliar to Alice stand out. California Institute

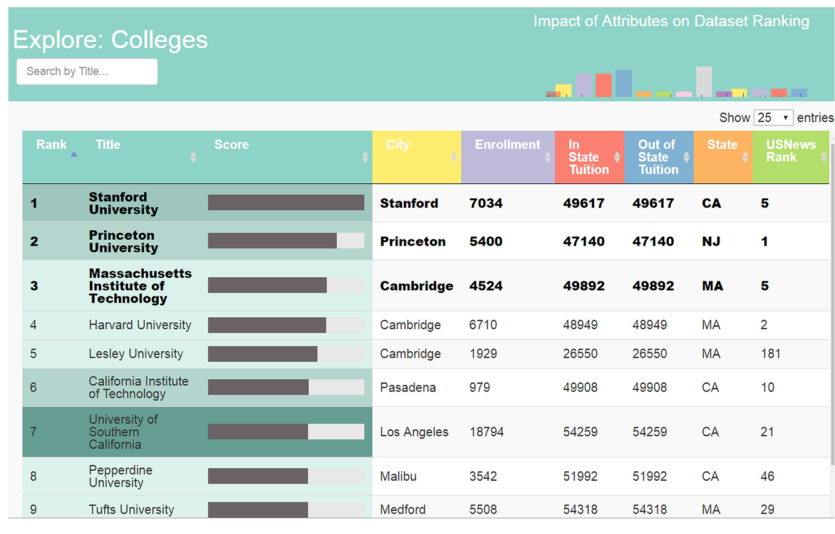

Figure 4: RanKit Explore view shows a ranking learned over the Colleges dataset.

of Technology and University of Southern California are ranked among the top ten colleges with high confidence values. Alice decides to spend additional time researching these schools to better inform her college application choice.

\section{CONCLUSION}

In this work we demonstrated RanKit, an interactive system for personalized rank building from user preferences. Important directions for future research include exploring the potential use of semi-supervised and active learning paradigms to improve the interactive ranking experience, as well as further developing visualizations for rank understanding.

\section{ACKNOWLEDGMENTS}

The authors would like to thank the Computing Resources Association for Women for support through the CREU program. This work was also partially funded by the Massachusetts High Technology Council, National Science Foundation IIS-1815866, and US Department of Education GAANN Fellowship P200A150306.

\section{REFERENCES}

[1] Ben Carterette, Paul N Bennett, Maxwell Chickering, and Susan T Dumais. 2008. Here or there. In European Conference on Information Retrieval. Springer, 16-27.

[2] Alex Endert, M Shahriar Hossain, Naren Ramakrishnan, Chris North, Patrick Fiaux, and Christopher Andrews. 2014. The human is the loop: new directions for visual analytics. Fournal of intelligent information systems 43, 3 (2014), 411-435.

[3] Samuel Gratzl, Alexander Lex, Nils Gehlenborg, Hanspeter Pfister, and Marc Streit. 2013. Lineup: Visual analysis of multi-attribute rankings. IEEE transactions on visualization and computer graphics 19, 12 (2013), 2277-2286.

[4] Thorsten Joachims. 2002. Optimizing search engines using clickthrough data. In Proceedings of the 8th ACM SIGKDD International Conference on Knowledge Discovery and Data Mining. ACM, 133-142.

[5] Caitlin Kuhlman and Elke Rundensteiner. 2017. Towards an Interactive Learn-toRank System for Economic Competitiveness Understanding. In KDD 2017 Interactive Data Exploration and Analytics Workshop.

[6] Tie-Yan Liu. 2009. Learning to rank for information retrieval. Foundations and Trends ${ }^{\circledR}$ in Information Retrieval 3, 3 (2009), 225-331.

[7] Emily Wall, Subhajit Das, Ravish Chawla, Bharath Kalidindi, Eli T Brown, and Alex Endert. 2018. Podium: Ranking data using mixed-initiative visual analytics. IEEE transactions on visualization and computer graphics 24, 1 (2018), 288-297.

[8] Meike Zehlike, Francesco Bonchi, Carlos Castillo, Sara Hajian, Mohamed Megahed, and Ricardo Baeza-Yates. 2017. Fa* IR: A Fair Top-k Ranking Algorithm. In Proceedings of the 2017 ACM on Conference on Information and Knowledge Management. ACM, 1569-1578. 